\title{
Papel do desbridamento e sinovectomia artroscópica no tratamento da rizartrose-experiência inicial do serviço
}

\author{
A, Ferreira, R. Mendes, A, Costa, P. Canela, R. Freitas \\ Serviço de Ortopedia e Traumatologia. Centro hospitalar de Vila Nova de Gaia/Espinho. Vila Nova de Gaia. Portugal.
}

Resumen: Objetivos:Apresentar a experiência inicial do serviço com o uso do desbridamento e sinovectomia artroscópica no tratamento da rizartrose.

Realizou-se um estudo retrospetivo de 10 doentes com rizartrose submetidos a desbridamento e sinovectomia artroscópica. Avaliou-se a dor através da escala visual analógica (EVA), o nível funcional com o questionário de QuickDASH, a força de pinça em relação à mão contralateral, o grau de satisfação subjetiva e as complicações no pós-operatório.

O score médio de QuickDASH no pré-operatório foi de 51,5 e no pós-operatório de 20,5 ( $p=0,036)$. Houve melhoria a nível da dor com uma avaliação pré operatória de 7,4 pontos para 2,6 pontos no pós-operatório $(p=0,012)$. Comparativamente ao lado contralateral, a média da força de pinça no pré operatório foi de $61,7 \%$ melhorando para $81 \%$ no pós-operatório $(p=0,067)$.A complicação mais grave no pós-operatório foi o desenvolvimento de um quadro de algodistrofia em um doente. Como em qualquer nova técnica, as indicações para o seu uso serão motivo de debate e evolução ao longo do tempo, o que se aplica ao trabalho apresentado em que algumas das indicações podem ser controversas.

Além disso, trata-se de uma amostra muito pequena e com um tempo de seguimento médio curto, pelo que as conclusões obtidas devem ser interpretadas com cautela.

Palabras claves: Rizartrose, artroscopia, desbridamento, sinovectomia, dor, força de pinça.

\begin{abstract}
Objectives: Present our initial experience using the arthroscopic debridement and synovectomy in the treatment of rizarthrosis.

We performed a retrospective study of 10 patients with rizarthrosis who underwent arthroscopic debridement and synovectomy. We assessed pain using a visual analog scale (VAS), functional level with the QuickDASH questionnaire, the pinch strength compared to the contralateral hand, the degree of satisfaction and complications.

The mean preoperative QuickDASH score was 51.5 and 20.5 postoperatively $(p=0.036)$. There was improvement in the pain with a preoperative assessment of 7.4 points to 2.6 points postoperatively $(p=0.012)$. Compared to the contralateral side, the mean pinch strength preoperatively was $61.7 \%$ improving to $81 \%$ postoperatively $(p=0.067)$.

The most serious complication in the postoperative period was the development of a framework for algodystrophy in a patient .

As with any new technique, indications for its use are subject to debate and evolution over time, which applies to the article presented in which some of the indications may be controversial.

Moreover, it is a very small sample and a short mean follow-up, so the conclusions should be interpreted with caution.
\end{abstract}

Key words: Rizarthrosis, arthroscopy, debridement, synovectomy, pain, pinch strength.

A articulação trapézio-metacarpiana é o segundo local mais frequentemente atingido por osteoartrose na mão.

Os avanços na tecnologia têm permitido o exame e o tratamento artroscópico das pequenas articulações, com mínima morbilidade.

A avaliação artroscópica da articulação trapézio-metacarpiana permite a visualização direta de todos os componentes articulares sem comprometer a integridade capsular, tratando as lesões por via artroscópica ou aberta de acordo com as alterações observadas ${ }^{1-3}$.
Está indicada em doentes cujo tratamento conservador falhoul,2.

As contraindicações da artroscopia trapézio-metacarpiana incluem alterações degenerativas escafo-trapezio-trapezoideias graves (estadio IV de Eaton),
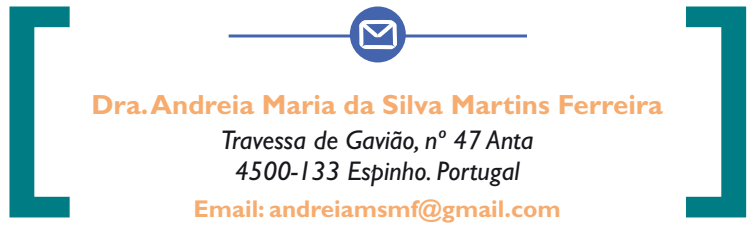


\begin{tabular}{c|c|c|}
\hline Estadio & \multicolumn{1}{c|}{ ALteRAções } \\
\hline I & Superfície articular intacta; rotura do ligamento dorsoradial; hipertrofia sinovial difusa. \\
\hline II & $\begin{array}{l}\text { Sinovite marcada. Destruição da cartilagem articular no } 1 / 3 \text { cubital da base do } 1^{0} \\
\text { metacarpiano e no } 1 / 3 \text { central da superfície distal do trapézio; rotura do ligamento } \\
\text { dorsoradial; laxidez do ligamento oblíquo anterior. }\end{array}$ \\
\hline III & $\begin{array}{l}\text { Perda de cartilagem generalizada em ambas as superfícies articulares; sinovite ligeira; } \\
\text { desgaste e laxidez dos ligamentos volares. }\end{array}$ \\
\hline Tabela. I. Classificação artroscópica da rizartrose segundo Badia ${ }^{2}$.
\end{tabular}

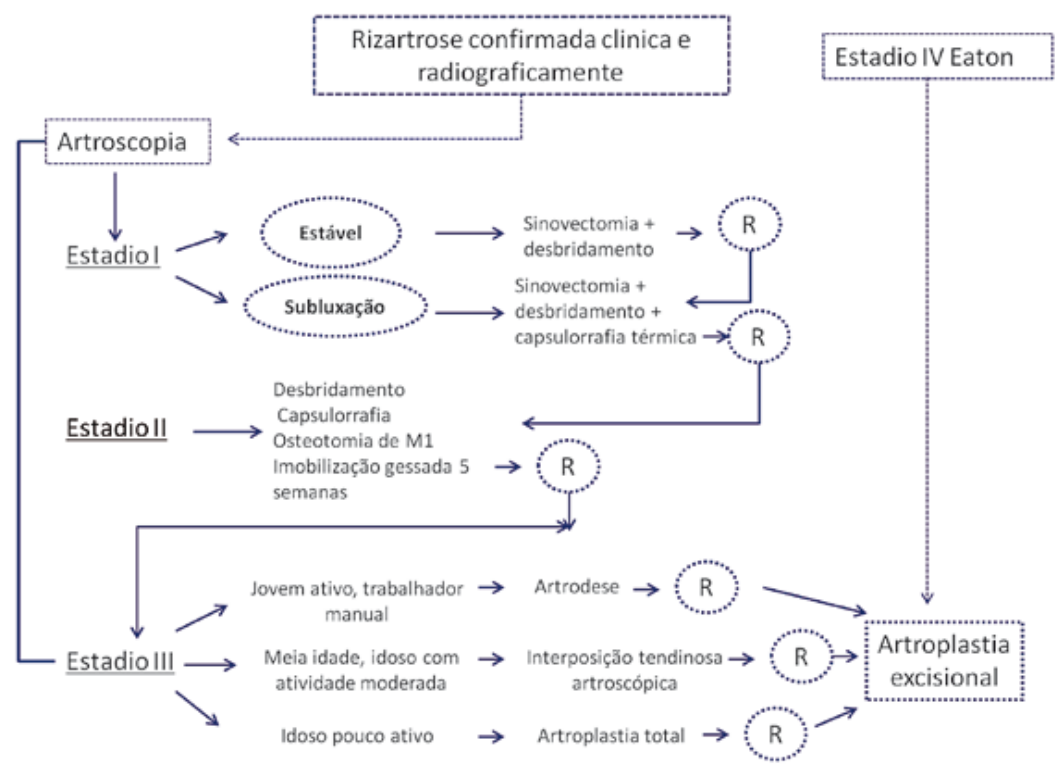

Figura. I. Algoritmo terapêutico proposto por Badia².

doentes idosos pouco ativos ou jovens trabalhadores manuais e doentes com contratura em adução ou subluxação grave do polegar ${ }^{1,2}$.

Uma nova classificação artroscópica foi proposta por Badia $^{2}$ e encontra-se descrita na (2) Tablea I.

Esta classificação permite obter um diagnóstico preciso e escolher um tratamento apropriado, o que permitiu a Badia desenvolver um algoritmo terapêutico ${ }^{1-4}$ a partir da mesma (2) Figura I.

\section{MATERIALY MÉTODO}

Os autores apresentam a experiência inicial do serviço com um estudo retrospetivo de 10 doentes com ri- zartrose (8 do sexo feminino e 2 do sexo masculino), submetidos a desbridamento e sinovectomia artroscópica. Todos os doentes tinham sido submetidos a tratamento conservador durante pelo menos 6 meses, que incluiu o uso de ortótose, AINE, fisioterapia e infiltração articular com corticóide.

Descreve-se sumariamente a técnica cirúrgica. Avaliou-se a dor no pré e pós- operatório, através da escala visual analógica (EVA), o nível funcional utilizando o questionário de QuickDASH no pré e pós-operatório, o grau de satisfação subjetiva e as complicações no pós-operatório.

A força de pinça foi medida em relação à mão contralateral, no pré e pós-operatório, utilizando o dinamómetro de Jamar. 


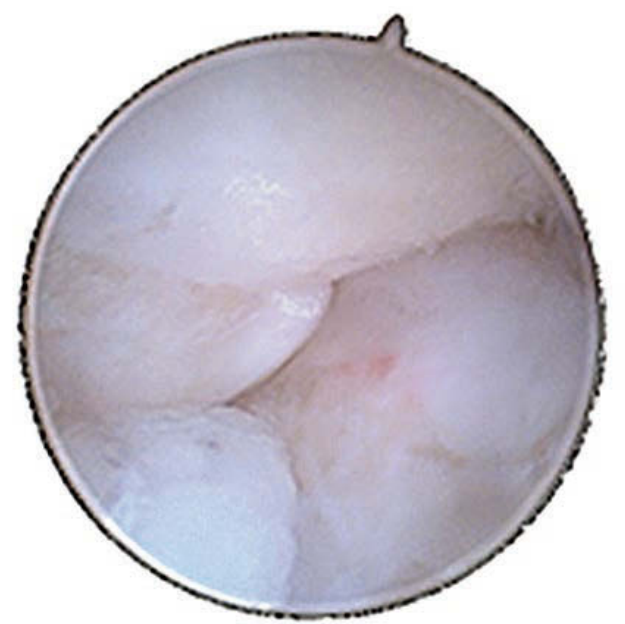

Figura 2. Imagem artroscópica demonstrando sinovite moderada da articulação

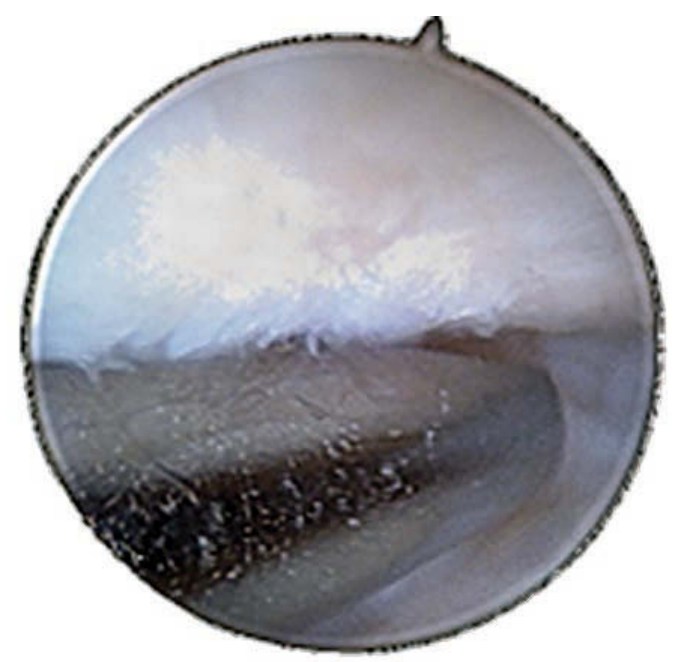

Figura 3. Fase inicial do desbridamento articular e sinovectomia.

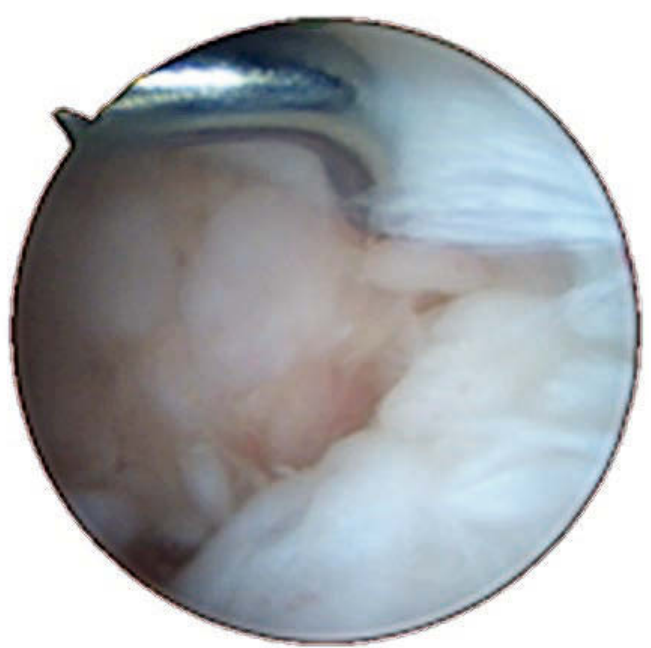

Figura. 4. Teste da integridade ligamentar.

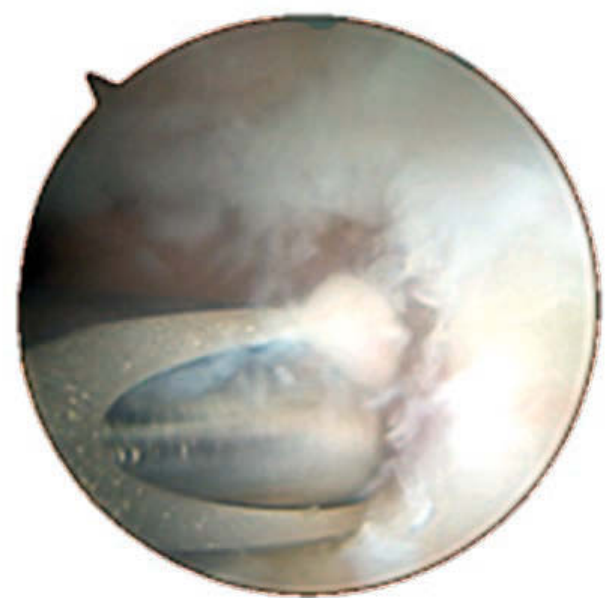

Figura 5. Fase final após desbridamento e sinovectomia.

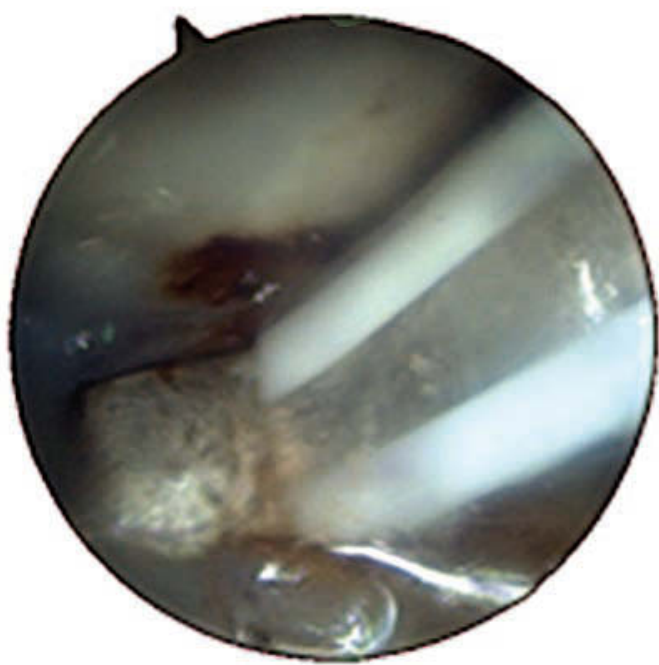

Figura 6. Termoretração cápsulo-ligamentar.

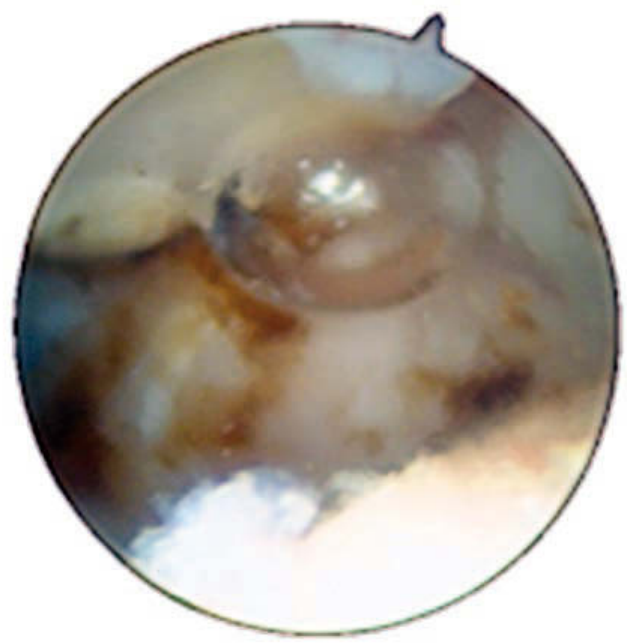

Figura 7. Aspecto final da articulação após sinovectomia, desbridamento e termoretração cápsulo-ligamentar. 


\section{Técnica cirúrgica}

É aplicado um garrote na raiz do braço e usada uma armadilha chinesa no polegar com $3 \mathrm{~kg}$ de tração longitudinal. Localiza-se a articulação por palpação e desenham-se as referências para depois se realizarem os portais: o portal I-R (radial), volar ao tendão do longo abdutor do polegar e o portal I -U (ulnar), no lado cubital do tendão do curto extensor do polegar.

A articulação é distendida com I a $3 \mathrm{ml}$ de soro fisiológico. Utilizou-se um artroscópio de $2,7 \mathrm{~mm}$ e $30^{\circ}$ de inclinação para visualização da articulação@ Figuras $2-7$.

\section{RESULTADOS}

Dos doentes operados, 8 eram do sexo feminino e 2 do sexo masculino. A idade média foi de 58,1 anos, com um mínimo de 48 anos e máximo de 66 anos. O tempo médio de seguimento foi de 10,6 meses (mínimo de 6 meses e máximo de 18 meses).

Radiologicamente, segundo a classificação de Eaton e Littler, 4 doentes apresentavam rizartrose grau II e 6 grau III.

Foram operadas 7 mãos esquerdas e 3 mãos direitas. De acordo com a classificação artroscópica de Badia², 4 doentes encontravam-se no estadio II e 6 doentes no estadio III.

Todos os doentes foram submetidos a desbridamento, sinovectomia e termoretração capsulo-ligamentar. No pós-operatório foi colocada imobilização gessada durante 4 semanas e utilização de ortótese noturna até aos 3 meses de pós-operatório.

O score médio de QuickDASH no pré-operatório foi de 51,5 e no pós-operatório de 20,5 ( $p=0,036)$. Houve melhoria a nível da dor com uma avaliação pré operatória de 7,4 pontos para 2,6 pontos no pós-operatório $(p=0,0 \mid 2)$.

Comparativamente ao lado contralateral, a média da força de pinça no pré operatório foi de 61,7\% melhorando para $81 \%$ no pós-operatório $(p=0,067)$.

A complicação mais grave no pós-operatório foi o desenvolvimento de um quadro de algodistrofia em um doente.

\section{DISCUSIÓN}

Até há relativamente pouco tempo, a avaliação clínica e o exame radiológico têm sido as únicas ferramentas disponíveis avaliar a rizartrose.

Em 1984 Eaton e Littler ${ }^{5}$ propuseram uma classificação de estadiamento radiográfico que tem sido amplamente aplicada. No entanto, é difícil fazer um diagnóstico preciso sobre a extensão da artrose baseando-se unicamente neste sistema de estadiamento.

A classificação artroscópica proposta por Badia ajuda a obter um diagnóstico mais preciso e a escolher $\mathrm{O}$ tratamento mais adequado ${ }^{2,3}$.

O tratamento da rizartrose por via artroscópica apresenta como vantagens a recuperação mais rápida, com menos cicatrizes e menos dor no pós-operatório ${ }^{6-8}$.

Segundo Badia, os benefícios da artroscopia na rizartrose são notórios principalmente nas fases iniciais da doençal, 2,4. Num estadio inicial, o doente apresenta queixas clínicas com radiografias normais, em resultado de sinovite ligeira a moderada. Estes doentes beneficiam de um desbridamento articular com sinovectomia e termoretração capsulo-ligamentar, nos casos de subluxação.

No nosso estudo, a maioria dos doentes apresentava doença num estadio radiológico e artroscópico mais avançado. De acordo com o algoritmo desenvolvido por Badial-4, doentes no estadio II e III são candidatos a gestos cirúrgicos adicionais nomeadamente osteotomia, artrodese ou artroplastia. Este algoritmo não foi criteriosamente seguido, o que poderá explicar o facto do grau de satisfação subjetiva ter sido inferior quando comparado com outros autores, ${ }^{9}$. Além disso, trata-se de uma técnica implementada recentemente no serviço com uma curva de aprendizagem exigente.

Existem poucos estudos na literatura que avaliam o papel do desbridamento e sinovectomia artroscópica no tratamento da rizartrose. $\bigcirc$ estudo de Furia ${ }^{9}$ que comparou os resultados do tratamento conservador com o desbridamento/sinovectomia artroscópica em doentes com rizartrose no estadio I e II, demonstrou melhoria da dor, nível funcional, força de pinça e satisfação pessoal nos doentes submetidos a tratamento cirúrgico. 
Os resultados obtidos no nosso trabalho são semeIhantes aos da literatura embora relativamente à força de pinça a diferença encontrada não é estatisticamente significativa.

Neste trabalho, o uso exclusivo do desbridamento e sinovectomia artroscópica teve como objectivo a evicção de gestos cirúrgicos mais agressivos (artroscópicos ou abertos) que, a longo prazo serão concerteza necessários já que neste caso as indicações cirúrgicas para o seu uso foram alargadas, podendo ser alvo de discussão.

Relativamente à termoretração capsulo-ligamentar, Badia recomenda o seu uso nos estadios I e II associados a instabilidade. Hofmeister et al, acreditam que o procedimento provoca adicionalmente uma neurectomia parcial da cápsula e tecidos moles, destruindo os receptores sensitivos aferentes e aliviando a dor. No entanto, esta teoria ainda não está comprovada." Existem vários estudos que relatam o uso de termoretração capsular no ombro mas a literatura é escassa relativamente ao seu uso no punho e mão. ${ }^{12,13}$ Embora os resultados possam ser superiores nestas articulações mais pequenas e não sujeitas a carga, não existem estudos que suportem esta teoria. Além disso, esta técnica pode causar lesões na superfície articular, nervos superficiais ou tendões e não está provado que evite a progressão da doença

As complicações associadas aos procedimentos descritos anteriormente incluem lesão do ramo sensitivo superficial do nervo radial ou da artéria radial, formação de hematoma, infeção e algodistrofia. Esta última complicação verificou-se em um dos doentes operados que tem melhorado progressivamente e mantém seguimento na consulta externa.

\section{CONCLUSIÓN}

A artroscopia tornou-se uma técnica fundamental no tratamento da patologia de grandes articulações. Atualmente as suas indicações foram alargadas para pequenas articulações, tal como a trapeziometacarpiana.

A avaliação artroscópica da rizartrose permite avaliar o grau de lesão articular e orientar o ortopedista na seleção do tratamento mais adequado.

Como em qualquer nova técnica, as indicações adequadas serão motivo de debate e evolução ao longo do tempo, o que se aplica ao trabalho apresentado em que algumas das indicações podem ser controversas. Além disso, trata-se de uma amostra muito pequena e com um tempo de seguimento médio curto, pelo que as conclusões obtidas devem ser interpretadas com cautela.

Desta forma, são necessários estudos clínicos adicionais para validá-la e incentivar a sua execução.

\section{CONFLICTOS DE INTERESES}

Os autores declaram não haver conflitos de interesse.

\section{BIBLIOGRAFÍA}

I. Badia A. Arthroscopy of the trapeziometacarpal and metacarpophalangeal joints. J Hand Surg Am. 2007;32A(5): 707-24.

2. Badia A. Trapeziometacarpal arthroscopy: a classification and treatment algorithm. Hand Clin. 2006;22:153-163.

3. Badia A, Khanchandani P. Treatment of early basal joint arthritis using a combined arthroscopic debridement and metacarpal osteotomy. Tech Hand Upper Extrem Surg. 2007; I I (2): I 68-73.

4. Badia A, Khanchandani P. Thumb carpometacarpal joint arthroscopy:a classification system and rationale for treatment. Oper Tech Orthop. 2007; 17(2): 125132.

5. Eaton RG, Lane LB, Littler JW, Keyser JJ. Ligament reconstruction for the painful thumb carpometacarpal joint: a long term assessment. J Hand Surg Am. 1984;9A:692-699.

6. Adams JE, Steinmann SP, Culp RW.Trapezium-sparing options for thumb carpometacarpal joint arthritis. Am J Orthop. 2008:37(8 suppl I):8-I I.

7. Croog AS, Rettig ME. Newest advances in the operative treatment of basal joint arthritis. Bull NYU Hosp Jt Dis. 2007;65(I):78-86.

8. Berger RA. A technique for arthroscopic evaluation of the first carpometacarpal joint. J Hand Surg Am. 1997;22A: 1077-1080.

9. Furia JP. Arthroscopic debridement and synovectomy for treating basal joint arthritis. Arthroscopy. 20I0;26(I);34-40. 
10. Culp RW, Rekant MS. The role of arthroscopy in evaluating and treating trapeziometacarpal disease. Hand Clin. 2001;17(2):315-9.

I I. Edwards SG, Osterman AL. Trapeziectomy with thermal capsular modification. In: Geissler W (Ed). Wrist arthroscopy. New York: Springer; 2005. p. I70-8I.
12. Darlis NA, Weisner RW, Sotereanos DG. Partial scapholunate ligament injuries treated with arthroscopic debridement and thermal shrinkage. J Hand Surg Am. 2005;30(5):908-14.

13. Shih J, Lee H. Monopolar radiofrequency electrothermal shrinkage of the scapholunate ligament. Arthroscopy. 2006;22(5):553-7. 